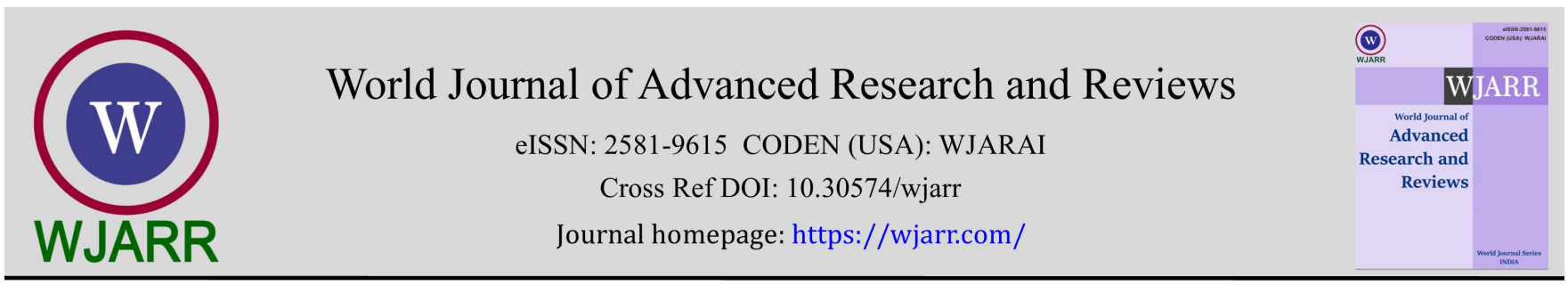

(RESEARCH ARTICLE)

Check for updates

\title{
Surgical evaluation and management of adnexal masses: A tertiary care center experience
}

\author{
Yasin Duran 1, Batuhan Üstün 2, *, Fırat Can Söğüt 2, Özlem Sevinç Ergül 2, R Ceren Avcı Çavdar 2 and \\ Mehmetbaki Şentürk ${ }^{2}$ \\ ${ }^{1}$ Namık Kemal University Hospital, Department of General Surgery, Tekirdağ, Turkey. \\ ${ }^{2}$ Namık Kemal University Hospital, Department of Gynecology and Obstetrics, Tekirdağ, Turkey.
}

World Journal of Advanced Research and Reviews, 2021, 09(01), 207-211

Publication history: Received on 07 January 2021; revised on 14 January 2021; accepted on 16 January 2021

Article DOI: https://doi.org/10.30574/wjarr.2021.9.1.0016

\begin{abstract}
Introduction: Intraoperative and postoperative outcomes of ovarian carcinoma patients who underwent cytoreductive surgery in our clinic has been evaluated with the measures of adjuvant chemotherapy requirement, recurrence and survival rates.
\end{abstract}

Materials and methods: Cases who underwent cytoreductive surgery between 2018-2019 in our clinic are evaluated in aspects of age at diagnosis, menopausal status, pathological stage of tumor, size of tumor, mode of surgical therapy, duration of surgery, neoadjuvant/adjuvant therapy choice, follow-up protocol, synchronous/metachronous state, rate of relapses and survival.

Results and discussion: The mean age of total 23 operated cases is $59 \pm 8.9 . \% 69,6$ of all cases is found to be primary ovarian carcinoma, whereas $\% 30,4$ is ovarian metastasis of other malignancies. Among metastatic cases, three is primary carcinoma of colon, one is gastric cancer and one is primary carcinoma of bladder. In other two cases, invasion of endometrial cancer to the ovary was observed. In addition to the standard surgical procedures, one case has undergone diagnostic mass resection, one sole total abdominal hysterectomy (TAH) and bilateral salpingooferectomy (BSO) and 21 omentectomy (OMT). Among these 21 cases one has undergone bilateral pelvic lymph node dissection and five $(\% 21,7)$ bilateral pelvic paraaortic lymph node dissection (BPPLND). In addition to TAH, BSO, OMT and BPPLND; nine $(\% 39,1)$ cases has undergone low anterior resection, one cystectomy, one distal pancreas resection and one partial gastrectomy. Three (\%13) of the cases has been diagnosed in stage 1 , one in stage 2 , nine (\%39,1) in stage 3 and ten $(\% 43,5)$ in stage 4 . Four $(\% 17,4)$ of the cases had taken neoadjuvant chemotherapy priorly to the surgery. Twenty-one $(\% 91,3)$ of the cases took adjuvant chemotherapy and one chemoradiotherapy. Relapses has been observed in the follow up of two $(\% 8,7)$ cases. Nineteen $(\% 82,6)$ of the cases are currently in disease-free state and four $(\% 17,4)$ has been in exitus state.

Conclusion: Surgery is the major modality of treatment in ovarian cancer. Various surgical resections by experienced gynecologists may be in need to achieve optimal cytoreduction and multidisciplinary approach is substantially important.

Keywords: Anterior resection; Ovarian tumor; Survival rate; Cytoreduction

\footnotetext{
* Corresponding author: Batuhan Üstün

Namık Kemal University Hospital, Department of Gynecology and Obstetrics, Tekirdağ, Turkey. 


\section{Introduction}

Among all the cancer types, ovarian cancers are seventh most frequent worldwide, second in developed countries and third in underdeveloped countries [1]. \%95 of ovarian cancers are originated from epithelial cells whereas the rest are from other cells of the organ. High grade serous carcinoma is the most frequently observed epithelial ovarian cancer [1]. Mean age of diagnosis is 63 and staging is made surgically. Due to the stealthy progressive nature of the disease, most of the cases are diagnosed in late stages; \%59 with a distant metastasis [1]. Secondary ovarian tumors develop with the metastasis of tumors of non-ovarian tissue origin. Primary therapeutical approach for ovarian cancer is surgery. Cytoreduction has the vast importance especially in the high stage diseases. Survival rates depend on the effect of the adjuvant therapies which rely on the success of the surgery mostly.

In this study, our aim is to retrospectively investigate the ovarian cancer cases we operate in our clinic.

\section{Material and methods}

In this retrospective study, patients who were operated for a mass in the ovary in our clinic between2018-2019 and were diagnosed with primary or secondary ovarian cancer pathologically were included. The study was approved by Tekirdağ Namık Kemal University Faculty of Medicine ethics committee decision dated 25.03.2020 and numbered 2020 58.03.08. Data is obtained by using the gynecology service follow-up files of the cases and accessing the contact information of the patients. These cases were evaluated in terms of age at the time of diagnosis, menopausal status, tumor stage, tumor size, surgical treatment method, duration of operation, whether neoadjuvant / adjuvant therapy was applied, follow-up protocol, secondary cancer association, recurrence and survival. Transvaginal ultrasonography, pelvic examination, tumor markers, cervical smear, endometrial sampling and pelvic magnetic resonance imaging were used in the preoperative evaluation of the cases, while mammography, colonoscopy, and endoscopy were used to investigate metastasis or secondary cancer. Hysterectomy, bilateral salpingo oophorectomy, pelvic / paraaortic lymph node dissection and omentectomy were performed as surgical treatment. Biopsy was taken from areas that seemed tumorally suspicious during exploration. Macroscopically visible tumoral areas were resected. All cytoreductions were done by a gynecologist. Intestinal anastomoses were performed by the general surgeon and the urinary diversions are done by the urologist. The materials obtained from the operations were evaluated by the pathology team of our hospital. The cases were staged according to FIGO 2009 (International Federation of Gynecology \& Obstetrics) [2] and AJCC (American Joint Committee on Cancer) [3] system. Patients were called for a check-up every three months in the first postoperative year. During the controls, magnetic resonance imaging with serum CA-125, CA15-3, CA19-9, CEA parameters was requested at each visit after a comprehensive physical examination, gynecological examination and a pelvic/transvaginal ultrasound examination.

Statistical analysis of the data has been made with SPSS (statistical package for social sciences) version 26 for Windows. Mean ( \pm ), standard deviation (std. deviation) and percentage (\%) were the descriptive statistical measures.

\section{Results and discussion}

The mean age of the cases was calculated as $59 \pm 8.9$ (Table 1). 95.7\% of the cases were in the postmenopausal period and $4.3 \%$ were in the premenopausal period. $69.6 \%$ were found as primary ovarian carcinoma, and $30.4 \%$ as secondary ovarian tumors.

Diagnostic mass resection in one case, total abdominal hysterectomy (TAH) and bilateral salpingooferectomy (BSO) in one case, TAH + BSO + omentectomy in two cases, TAH + BSO + bilateral pelvic lymph node dissection + omentectomy in one case, $\mathrm{TAH}+\mathrm{BSO}+$ bilateral pelvic paraaortic lymph node dissection (BPPLND) + omentectomy in five cases $(21.7 \%), \mathrm{TAH}+\mathrm{BSO}+\mathrm{BPPLND}+$ omentectomy + low anterior resection in nine cases (39.1\%), TAH + BSO + BPPLND + omentectomy + ureter resection in one case, $\mathrm{TAH}+\mathrm{BSO}+\mathrm{BPPLND}+$ omentectomy + cystectomy in one case, TAH + BSO + BPPLND + omentectomy + distal pancreatic resection in one case, and TAH + BSO + BPPLND + omentectomy + partial gastrectomy in one case has been performed (Table 1)

Three $(13 \%)$ of the cases were diagnosed in stage 1 , one in stage 2 , nine $(39.1 \%)$ in stage 3 , and ten (43.5\%) in stage 4 . Four (17.4\%) of the operated patients received neoadjuvant treatment and 19 (23\%) did not receive neoadjuvant treatment. 21 (91.3\%) patients received chemotherapy and one patient received chemoradiotherapy as adjuvant therapy. One continued his outpatient clinic controls without receiving adjuvant therapy. Recurrence was detected in the follow-up of two (8.7\%) patients. Nineteen (82.6\%) of the patients were continuing their follow-up in our clinic, and four patients $(17.4 \%)$ died. 
Table 1 Variety and proportions of cases

\begin{tabular}{|l|l|l|l|l|}
\hline \multicolumn{2}{|c|}{ Count } & Percentage & Valid percentage & Cumulative percentage \\
\hline Diagnostic mass resection & 1 & 4,3 & 4,3 & 4,3 \\
\hline TAH BSO & 1 & 4,3 & 4,3 & 8,7 \\
\hline TAH BSO omentectomy & 2 & 8,7 & 8,7 & 17,4 \\
\hline $\begin{array}{l}\text { TAH BSO pelvic lymphadenectomy } \\
\text { and/or omentectomy }\end{array}$ & 1 & 4,3 & 4,3 & 21,7 \\
\hline $\begin{array}{l}\text { TAH BSO PPLND and/or } \\
\text { omentectomy }\end{array}$ & 5 & 21,7 & 21,7 & 43,5 \\
\hline $\begin{array}{l}\text { TAH BSO PPLND and/or } \\
\text { omentectomy and low anterior } \\
\text { resection }\end{array}$ & 9 & 39,1 & 39,1 & 82,6 \\
\hline $\begin{array}{l}\text { TAH BSO PPLND and/or } \\
\text { omentectomy and ureter restion }\end{array}$ & 2 & 8,7 & 8,7 & 91,3 \\
\hline $\begin{array}{l}\text { TAH BSO PPLND and/or } \\
\text { omentectomy and cystectomy }\end{array}$ & 1 & 4,3 & 4,3 & 95,7 \\
\hline $\begin{array}{l}\text { TAH BSO PPLND and/or } \\
\text { omentectomy and distal pancreas } \\
\text { resection }\end{array}$ & 1 & 4,3 & 4,3 & 100,0 \\
\hline Total & 23 & 100,0 & 100,0 & \\
\hline
\end{tabular}

When we examine the follow-up periods, seven of our cases were operated between September and December 2018 and have been followed up for two years. Our four patients who died were operated in the same date range. Four of our patients were operated between January and May 2019 and completed the 15th month in their follow-up. Eight of our cases were operated between June and December 2019 and applied to us for the first twelve months of follow-up.

Considering the postoperative complications in our cases; in one patient, evisceration from the sub-umbilical incision line was detected on postoperative fifteenth day, and repair was performed with general surgery. No pathology was observed after revision surgery.

Serous endometrial cancer ovarian metastasis was detected in a second case, and swelling was detected in the legs in the sixth postoperative month. She was hospitalized with a diagnosis of deep vein thrombosis. Since deep vein thrombosis was not considered in the imaging performed, the patient whose clinic recovered was discharged with thrombolytic therapy at prophylaxis dose.

In a third case, the patient was hospitalized after the vaginal cuff sutures were opened in the second postoperative week. Sterile dressing was applied. Upon detection of abscess, it was drained regularly with a sterile aspirator. The patient, who was cared for in the hospital for 16 days, was discharged after her general condition improved.

In our fourth case, after the incisional hernia was detected in the seventh month postoperatively, he was referred to general surgery. The patient, who was not considered for operation, continues her routine follow-up.

When we evaluate the reasons for exitus; one of the patients was hospitalized in the intensive care unit after massive transfusion due to operative bleeding in the postoperative period. She died after transfusion-related lung injury (TRALI) during follow-up.

The second patient was diagnosed with ovarian metastasis of ring cell gastric cancer. She refused to accept treatment after the diagnosis and died due to respiratory arrest upon widespread systemic metastasis of the tumor. 
Our third patient died after a cerebrovascular event due to not using the antithrombotic treatment prescribed regularly in the postoperative period.

Our last patient died after a cardiac arrest due to the development of pulmonary embolism and subsequent increase in respiratory distress in the thirteenth month after the operation.

The most important step in the treatment of advanced stage ovarian cancer is cytoreductive surgery. The aim in surgery should be to reach R0, all regions with macroscopic tumors should be resected. Ovarian cancers progress insidiously, do not show symptoms in the early stages, so they are usually diagnosed in the late stages [4]. Among the prognostic criteria, postoperative residual tumor is one of the most important. The less tumor burden remaining after surgery, the better the response to treatment and the increased survival [5]. In line with this information, applications such as bowel resection, urinary system resections, partial pancreatectomy, partial gastrectomy, total omentectomy, peritoneal stripping have been surgeries accompanying hysterectomy and oophorectomy in advanced ovarian cancers.

Intestines are one of the organs in which ovarian cancers frequently metastasize. It may directly invade the rectum or sigmoid colon. Primary or secondary colon cancer was detected in nine of our cases accompanying the ovarian tumor. Considering the possibility of permanent or temporary colostomy for complete cytoreduction in these cases, radical colon resections were performed. In a multicenter study conducted by Miseon Kim et al., segmental resection and tumor resection were compared as surgical methods in ovarian cancer cases, and it was stated that segmental resection was more beneficial in terms of prognosis in advanced cases. It was stated that there was no difference between tumor resection and segmental resection in stage I-II cases [6].

In the study of Philipp Harter et al.; it was stated that patients who underwent bowel resection, diaphragm resection, splenectomy or liver resection and therefore been provided optimal cytoreduction, total survival rates are increased compared to patients who underwent standard surgery [7].

In a study by Dennis S Chi et al., It was stated that progression-free survival and overall survival increased in patients who underwent surgery for optimal cytoreduction with the inclusion of upper abdominal organs in advanced stage epithelial ovarian cancers and whose residual tumor diameter was $<1 \mathrm{~cm}$ compared to patients who underwent standard surgery[8]. In addition, it was emphasized that primary cytoreduction performed by specialists experienced in gynecological oncology is important in reaching R0 and having less complications. It has been stated by the general surgery specialists who were invited to the operation that, especially in cases with advanced stage and conglomerated pelvic region, are often considered inoperable. On the other hand, the legal responsibilities of bowel anastomoses after resection are on general surgery specialists, and this is the case for our country.

In a study by Nicolae Bacalbasa et al., it was discussed that colon resection for complete cytoreduction in cases with colorectal involvement secondary to ovarian cancer was discussed [9]. Although complications of colorectal surgery have been encountered, it has been concluded that these surgeries should be performed in a well-equipped center due to increased survival. The survival rate was found to be $81 \%$ in follow-up, and considering the stage of the disease, it can be said that complete cytoreductive surgery has a positive effect on survival.

Esra Özbaşlı's study mentioned the importance of palpable lymph node dissection in advanced ovarian tumors [10]. It has been discussed that removing palpable lymph nodes is sufficient for cytoreduction, and radical pelvic paraaortic lymph node dissection has no effect on survival. In our cases, when it was detected that they were in advanced stage, total lymph node dissection was not performed, and macroscopic palpable nodes were removed.

\section{Conclusion}

Surgery is shown to be the most effective modality of therapy of all among chemotherapy, radiotherapy and surgery for treatment of high stage ovarian cancers. When the complications of the ovarian cancer surgery are also taken into account, it will be right to emphasize the importance of the need of a fully equipped center with experienced surgeons for this option. Positive responses to adjuvant therapies with better survival rates increase with optimal cytoreductive surgeries. 


\section{Compliance with ethical standards}

\section{Acknowledgments}

The authors would like to thank the staff of Namık Kemal University Hospital archive for their help during the collection of the data of this study.

\section{Disclosure of conflict of interest}

There are no conflicts of interest.

\section{Statement of informed consent}

No informed consent was needed during the study as it is a retrospective analysis.

\section{References}

[1] Bostancı E, Ayas S, Boza A, Gürbüz A, Karateke A. Retrospective Analysis Of 53 Cases With Borderline Ovarian Tumors. Med J Bakirkoy. 2014; 10(1): 27-32.

[2] Prat J. FIGO's staging classification for cancer of the ovary, fallopian tube, and peritoneum: Abridged republication. Journal of Gynecologic Oncology. 2015; 26(2): 87.

[3] Egner JR. AJCC Cancer Staging Manual. JAMA. 2010; 304(15): 1726-1727.

[4] Ebell M. H., Culp, M. B., \& Radke T. J. (2016). A Systematic Review of Symptoms for the Diagnosis of Ovarian Cancer. American journal of preventive medicine, 50(3), 384-394.

[5] Shih KK, Chi DS. Maximal cytoreductive effort in epithelial ovarian cancer surgery. J Gynecol Oncol. 2010; 21(2): 75-80.

[6] Kim M, Suh DH, Park J, Paik ES, Lee, et al. Survival impact of low anterior resection in patients with epithelial ovarian cancer grossly confined to the pelvic cavity: A Korean multicenter study. Journal of Gynecologic Oncology. 29(4).

[7] Harter P, Muallem ZM, Buhrmann C, et al. Impact of a structured quality management program on surgical outcome in primary advanced ovarian cancer. Gynecol Oncol. 2011; 121(3): 615-619.

[8] Chi DS, Eisenhauer EL, Zivanovic 0, et al. Improved progression-free and overall survival in advanced ovarian cancer as a result of a change in surgical paradigm. Gynecol Oncol. 2009; 114(1): 26-31.

[9] Bacalbasa N, Balescu I, Dima S. Rectosigmoidian Involvement in Advanced-stage Ovarian Cancer - Intraoperative Decisions. In Vivo. 2017; 31(5): 973-977.

[10] Özbaşlı E. İleri evre epitelyal over kanserinde lenf nodu diseksiyonunun sağkalıma etkisi. Uzmanlık tezi. (Thesis of specialty training) Zeynep Kamil Kadın ve Çocuk Hastalıkları Eğitim ve Araştırma Hastanesi. 2009. 